\title{
Throat Carriage Rate and Antimicrobial Resistance of Streptococcus pyogenes In Rural Children in Argentina
}

\author{
Gastón Delpech', Mónica Sparo ${ }^{1,2}$, Beatriz Baldaccini', Gisela Pourcel', Sabina Lissarrague², Leonardo García Allende ${ }^{3}$ \\ ${ }^{1}$ School of Medicine, Universidad Nacional del Centro de la Provincia de Buenos Aires, Olavarría; ${ }^{2}$ Hospital Ramón Santamarina, Tandil; ${ }^{3}$ Center of \\ Biochemical Studies, Tandil, Argentina
}

Objectives: The aim of this study was to determine the prevalence of asymptomatic carriers of group A $\beta$-hemolytic streptococci (GAS) in children living in a rural community and to investigate the association between episodes of acute pharyngitis and carrier status.

Methods: Throat swabs were collected from September to November 2013 among children 5-13 years of age from a rural community (Maria Ignacia-Vela, Argentina). The phenotypic characterization of isolates was performed by conventional tests. Antimicrobial susceptibility was assayed for penicillin, tetracycline, chloramphenicol, erythromycin, and clindamycin (disk diffusion). The minimum inhibitory concentration was determined for penicillin, cefotaxime, tetracycline, and erythromycin.

Results: The carriage of $\beta$-hemolytic streptococci was detected in $18.1 \%$ of participants, with Streptococcus pyogenes in 18 participants followed by $S$. dysgalactiae ssp. equisimilis in 5 . The highest proportion of GAS was found in 8 to 10 -year-old children. No significant association between the number of episodes of acute pharyngitis suffered in the last year and the carrier state was detected $(p>0.05)$. Tetracycline resistance $(55.5 \%)$ and macrolide-resistant phenotypes (11.1\%) were observed. Resistance to penicillin, cefotaxime, or chloramphenicol was not expressed in any streptococcal isolate.

Conclusions: The present study demonstrated significant throat carriage of GAS and the presence of group C streptococci (S. dysgalactiae ssp. equisimilis) in an Argentinian rural population. These results point out the need for continuous surveillance of GAS and non-GAS carriage as well as of antimicrobial resistance in highly susceptible populations, such as school-aged rural children. An extended surveillance program including school-aged children from different cities should be considered to estimate the prevalence of GAS carriage in Argentina.

Key words: Streptococcus pyogenes, Prevalence, Pharyngitis, Rural population, Argentina

\section{INTRODUCTION}

Streptococcus pyogenes (group A $\beta$-hemolytic streptococci, GAS) is a re-emerging pathogen worldwide and one of the

Received: December 17, 2015 Accepted: December 18, 2016

Corresponding author: Mónica Sparo, PhD

1406 Gral Paz, Tandil 7000, Argentina

Tel: +54-249-442-2011, Fax: +54-249-442-8797

E-mail:monicasparo@gmail.com

This is an Open Access article distributed under the terms of the Creative Commons Attribution Non-Commercial License (http://creativecommons.org/licenses/bync/4.0// which permits unrestricted non-commercial use, distribution, and reproduction in any medium, provided the original work is properly cited. biggest challenges for public health. Most commonly, GAS infections are observed in the pharynx. Children between five and seven years of age are more susceptible, with seasonal patterns in occurrence. Human skin and mucous membranes are their only known natural reservoirs. Transmission occurs through asymptomatic nasopharyngeal carriers [1]. The clinical manifestations range from superficial to invasive disease, which may present with clinical manifestations associated with toxin production and high mortality rates [2].

GAS remains sensitive to penicillin, the antimicrobial of choice in the empirical treatment of streptococcal infections. Macrolides are a therapeutic alternative for patients allergic to 
penicillin. Macrolide resistance develops due to their use in clinical practice, and has become a worldwide emerging problem [3]. One of the mechanisms of macrolide resistance is inducible $\left(_{i}\right)$ or constitutive $\left({ }_{c}\right)$ dimethylation of 235 ribosomal RNA (the $M L S_{B}$ phenotype). Additionally, the existence of an active efflux mechanism ( $M$ phenotype) has been described. The most common mechanism of tetracycline resistance is ribosomal protection. Chloramphenicol resistance occurs through drug inactivation by acetylation [4]. In Argentina, resistant penicillin strains have not been detected, and a prevalence of tetracycline resistance lower than $10 \%$ has been reported. Furthermore, in Argentina, an increase in erythromycin resistance has been observed, with the M phenotype as the predominant mechanism [5].

The asymptomatic carriage of GAS in the population of school-aged children is a frequent and dynamic event. Colonized children can eliminate GAS, remain carriers, or develop an infectious disease. Although colonization usually is harmless for pediatric carriers, they could transmit GAS to others. Throat carriage has gained great importance due to the reemergence of severe diseases produced by this bacterium [6].

Worldwide, each year, approximately $15 \%$ of school-aged children are estimated to have GAS pharyngitis [7]. The County Health System of Tandil (Buenos Aires Province, Argentina) reported a significantly greater prevalence in the rural area of Maria Ignacia-Vela. Rural pediatric carriage may constitute a GAS reservoir associated with increases of acute pharyngitis episodes.

The aim of this study was to determine the prevalence of asymptomatic carriers of GAS in children living in a rural community and to investigate the association between episodes of acute pharyngitis and carrier status.

\section{METHODS}

\section{Study Design (Observational, Prospective, and Transversal Research)}

\section{Collection and initial processing of samples}

Throat swabs were collected from September to November 2013 among children from the rural community of María Ignacia-Vela, Tandil County, Argentina.

Briefings with parents or guardians were carried out and written informed consent was obtained. This research was approved by the Ethics Committee, Hospital de Clínicas, Argentina.

Children between 5 and 13 years of age, without acute pharyngitis, who had not received antimicrobials for four weeks and who had not been hospitalized for 3-month prior to obtaining the samples were included as participants.

The appropriate ethical regulations (Declaration of Helsinki and Argentinian laws) were applied. Results were analyzed in an anonymous fashion, ensuring the confidentiality of the information that was obtained.

A total of 138 participants were considered, but after several meetings with the goal of including as much of the pediatric population as possible, nine children were eliminated because the parents did not agree to participate and two refused to be included. A total of 127 children 5-13 years of age, with a mean age of $10.40 \pm 3.19$ years, were included. Participants were separated into three age groups. Group I included children 5-7 years of age; group II, children 8-10 years of age; and group III, children 11-13 years of age. Children with GAS in their throats without signs or symptoms of acute tonsillitis at the time of examination were considered to be asymptomatic carriers.

Throat swab samples were inoculated onto Columbia agar with $5 \%$ sheep blood and incubated at $35^{\circ} \mathrm{C}$ in $5 \%$ carbon dioxide for 24 hours.

\section{Phenotypic characterization of bacterial isolates}

The phenotypic characterization of bacterial isolates to the species level was carried out by conventional tests and the detection of Lancefield-group antigens (BioMérieux, Buenos Aires, Argentina), following the manufacturer's instructions [2]. The quality controls were S. pyogenes ATCC 19615 and S. agalactiae ATCC 13813.

The VITEK $2^{\circledR}$ Compact automated system (BioMérieux) was used for the validation of the phenotypic characterization.

Each isolate was assigned a co-relative alphanumerical identification code that included the sampling date. An electronic record was maintained in an Excel ${ }^{\mathrm{TM}}$ file.

\section{Antimicrobial susceptibility in vitro tests}

Qualitative antimicrobial susceptibility was investigated by the disk diffusion method, as recommended by the Clinical and Laboratory Standards Institute (CLSI) [8]. The antimicrobial disks used were penicillin (10 IU), tetracycline $(30 \mu \mathrm{g})$, and chloramphenicol $(30 \mu \mathrm{g})$.

For detection of macrolide and lincosamide resistance phenotypes, the double-disk method was used, placing erythromycin $(15 \mu \mathrm{g})$ and clindamycin $(2 \mu \mathrm{g})$ disks closely together, at $15 \mathrm{~mm}$ from edge to edge, in the same Mueller-Hinton agar 
Table 1. Prevalence of $\beta$-hemolytic streptococci among children 5-13 years of age in the rural community of Maria Ignacia-Vela, Tandil, Argentina

\begin{tabular}{lrr}
\hline Throat culture & n & $\%$ \\
\hline GAS & 18 & 14.2 \\
Group C & 5 & 3.9 \\
Total $(n=127)$ & 23 & 18.1
\end{tabular}

GAS, group A $\beta$-hemolytic streptococci (Streptococcus pyogenes); Group C, S. dysgalactiae ssp. equisimilis.

plate with 5\% sheep blood [8].

Quantitative susceptibility was studied by the agar dilution method, according to the recommendations of CLSI [8]. The minimum inhibitory concentration (MIC) for penicillin, cefotaxime, tetracycline, and erythromycin were determined. When an isolate expressed resistance to more than two antimicrobial groups, it was considered to be multidrug resistant.

\section{Statistical Analysis}

Data analysis was performed using SPSS version 11.5 (SPSS Inc., Chicago, IL, USA). Descriptive measures (mean \pm standard deviation) were used for quantitative variables, and frequencies were used to present qualitative variables. The chi-square test was used for verifying the existence of associations. Odds ratios (ORs) with $95 \%$ confidence intervals were used for risk quantification, and the $t$-test for independent samples was used to compare mean values. The $p$-values $<0.05$ were considered to indicate statistical significance.

\section{RESULTS}

\section{Group A $\beta$-hemolytic Streptococci (Streptococ- cus pyogenes) Prevalence}

In this study, carriage of $\beta$-hemolytic streptococci was detected (18.1\%) among 5 to 13 -year-old children from a rural population. Table 1 shows the distribution of bacterial isolates according to the phenotypic characterization. GAS was the most prevalent type $\beta$-hemolytic streptococci found $(n=18)$, followed by group $C \beta$-hemolytic streptococci $(n=5)$.

The distribution of GAS carriers and non-carriers by age group is shown in Table 2 .

The highest percentage of GAS was found in children from group II (55.6\%), followed by participants from groups I (38.9\%) and III (5.5\%). GAS isolates were recovered from male (55.6\%) and female (44.4\%) children. In all groups, differences between female and male carriage were detected, with fe-
Table 2. Prevalence of group A $\beta$-hemolytic streptococci (Streptococcus pyogenes) by age group among children 5-13 years of age in the rural community of Maria Ignacia-Vela, Tandil, Argentina

\begin{tabular}{lcrrr}
\hline Group & Age (y) & $\mathbf{n}_{\mathbf{n c}}$ & $\mathbf{n}_{\mathbf{c}}(\mathbf{f} / \mathbf{m})$ & \% $_{\mathbf{c}}$ \\
\hline I & $5-7$ & 40 & $7(4 / 3)$ & 5.5 \\
II & $8-10$ & 31 & $10(4 / 6)$ & 7.9 \\
III & $11-13$ & 38 & $1(0 / 1)$ & 0.8 \\
Total (n=127) & & 109 & 18 & 14.2 \\
\hline
\end{tabular}

nc, non-carrier; c, carrier; f, female; $m$, male.

Table 3. Episodes of acute pharyngitis among children 5-13 years of age in the rural community of Maria Ignacia-Vela, Tandil, Argentina

\begin{tabular}{lcc}
\hline \multirow{2}{*}{ Culture } & \multicolumn{2}{c}{ Acute pharyngitis episodes } \\
\cline { 2 - 3 } & $\mathbf{2} \mathbf{2}^{\mathbf{1}}$ & Total \\
\hline GAS-negative & $21(19.3)$ & 109 \\
GAS-positive & $4(22.2)$ & 18 \\
Total & $25(19.7)$ & 127 \\
\hline
\end{tabular}

Values are presented as number (\%).

GAS, group A $\beta$-hemolytic streptococci (Streptococcus pyogenes).

${ }^{1}$ Number of episodes.

male predominance in group I (57.1\%). Male patients positive for GAS carriage were more common than female carriers in groups II (60\%) and III (100\%).

In Table 3, the culture results are shown for GAS and number of patients with $\geq 2$ episodes of acute pharyngitis.

In this study, no significant association was found between the number of episodes of acute pharyngitis suffered in the last year and carrier status $(p>0.05)$. According to these results, carrier children had 1.1 times the risk of having $\geq 2$ episodes of acute pharyngitis than those who did not carry GAS $(\mathrm{OR}, 1.1)$. It is important to note that most of the samples were not positive for GAS.

\section{Antimicrobial Susceptibility}

All GAS isolates were susceptible to cell-wall active agents, such as the $\beta$-lactam penicillin (MIC $\leq 0.06 \mu \mathrm{g} / \mathrm{mL}$ ) and a thirdgeneration cephalosporin, cefotaxime (MIC $\leq 0.06 \mu \mathrm{g} / \mathrm{mL}$ ).

Chloramphenicol-resistant GAS were not recovered from participants. Nevertheless, resistance to three of the assayed antimicrobials was expressed among human S. pyogenes isolated from this rural population.

Tetracycline resistance was observed in 10 of the 18 (55.5\%) studied GAS isolates. This resistance phenotype was the most prevalent among non-susceptible streptococci. Furthermore, 
the MIC range for tetracycline was the widest, ranging from 1 to $\geq 16 \mu \mathrm{g} / \mathrm{mL}$.

In 2 of the $18(11.1 \%)$ samples of S. pyogenes, macrolide resistance phenotypes were detected.

GAS-007 expressed a ${ }_{C} M L S_{B}$ phenotype, with high-level erythromycin resistance (MIC, $256 \mu \mathrm{g} / \mathrm{mL}$ ) and resistance to clindamycin.

Erythromycin resistance (MIC, $8 \mu \mathrm{g} / \mathrm{mL}$ ) and clindamycin susceptibility were observed in isolate GAS-018 (M phenotype).

\section{DISCUSSION}

In this study, $18.1 \%$ of the children were carriers of $\beta$-hemolytic streptococci, mostly characterized as GAS (14.2\%), as well as S. dysgalactiae spp. equisimilis to a lesser extent (3.9\%).

Carapetis et al. [7] showed that GAS carriage prevalence decreased with age, but in this study, the highest prevalence occurred in children 8-10 years of age (group II, 7.9\%), and a significantly lower prevalence was found in older children (group III, 0.8\%).

In the present study, a rate of global throat GAS carriage of $14.2 \%$ was found. Similarly, Takeuchi et al. [9], in a study of school-aged children, reported a rate of $12.2 \%$. However, the Argentinian Society of Pediatrics established the prevalence to be around 5 to 7\% [10]. According to Kreikemeyer et al. [11], carrier status, relapses, and recurrent infections by GAS can be explained by the same mechanism. Additionally, being a healthy carrier has been proposed to facilitate recurrent acute infections. Nevertheless, in the present study, no relationship was found between colonization and the number of episodes of acute pharyngitis suffered in the last year.

In this study, carriage prevalence was higher among children between 8 and 10 years of age (group II), followed by 5 to 7-year-old participants (group I), while a lower prevalence was detected for the age range of 11-13 years (group III). In MorocCo, a pediatric and adult population study showed that one of the two peaks of GAS prevalence was found among participants with a similar age range to group III, unlike what was observed in this study [12]. In Argentina, a study of GAS frequency in the throats of symptomatic pediatric patients showed that children between 5 and 10 years old were positive at a higher rate than those aged 11-13 years [13].

Antimicrobial resistance is an important concern for many bacterial pathogens. Specifically, GAS can cause invasive infections, making it important to monitor the emergence of resis- tant strains.

In GAS isolates, resistance to $\beta$-lactams such as penicillin and cefotaxime was not detected. In other countries, resistance to $\beta$-lactams has likewise not been observed. A multicenter study that included 25 institutions from eight European countries showed that all the investigated GAS isolates did not express resistance to penicillin or cefotaxime, in agreement with the results of this research [14].

In addition, resistance to chloramphenicol was not observed. In agreement with this result, previous Argentinian studies have not reported the recovery of chloramphenicol-resistant $S$. pyogenes $[2,15]$. In 188 streptococcal isolates recovered from children with pharyngitis in China, chloramphenicol resistance was not detected in any of the studied GAS isolates [16].

These GAS isolates from rural children presented a high prevalence of tetracycline resistance (55.5\%). In an Argentinian multicenter study, a lower frequency of tetracycline resistance (7.3\%) was observed among invasive and non-invasive S. pyogenes [2]. The prevalence of tetracycline-resistant GAS found in this study is significant, since previous reports have established tetracycline resistance as a relevant cofactor in the selection of erythromycin resistance $[17,18]$. A study in children from China reported a very high prevalence of tetracycline resistance among GAS isolates (92\%), almost twice the frequency observed in this study [16].

In $1.1 \%$ of GAS isolates from the studied region, erythromycin resistance was observed, expressed as two different macrolide-resistant phenotypes, $\mathrm{M}$ and $\mathrm{CMLS}_{\mathrm{B}}$. In Chinese pediatric patients, high levels of resistance to erythromycin (94.0\%) and clindamycin (96.9\%) were detected. These frequencies were significantly higher than those reported in this study, indicating the predominance of the ${ }_{C} M L S_{B}$ phenotype [19]. The $M L S_{B}$ phenotype was also the most prevalent among GAS isolates from pediatric patients in 8 European countries, while MLS phenotypes as well as a lesser frequency of $\mathrm{M}$ phenotype strains were observed in French children [14,20].

In 2004, in Argentina, a study that included nationwide reports assessing antimicrobial resistance in GAS isolates concluded that there were two prevalent macrolide resistance phenotypes among circulating streptococcal strains, the $M$ and ${ }_{i} M L S_{B}$ phenotypes, although the $M$ phenotype was not considered to be frequent [15].

The present study showed significant throat carriage of GAS and the presence of group C streptococci (S. dysgalactiae ssp. equisimilis) in an Argentinian rural pediatric population. 
These results underscore the need for continuous surveillance of the carriage of GAS and other $\beta$-hemolytic streptococci, as well as of antimicrobial resistance in highly susceptible populations, such as school-aged rural children. An extended surveillance program including school-aged children from different cities should be considered to assess the situation in Argentina.

\section{ACKNOWLEDGEMENTS}

We acknowledge the community of Maria Ignacia-Vela for their kind collaboration with this study, and the Alberto J. Roemmers Foundation (Argentina) for providing funding.

\section{CONFLICT OF INTEREST}

The authors have no conflicts of interest associated with the material presented in this paper.

\section{ORCID}

Gastón Delpech http://orcid.org/0000-0002-9109-7615

Monica Sparo http://orcid.org/0000-0002-0768-894X

Beatriz Baldaccini $h t t p: / / o r c i d . o r g / 0000-0002-1539-6947$

Gisela Pourcel http://orcid.org/0000-0002-5095-2160

Sabina Lissarrague http://orcid.org/0000-0002-4041-0931

Leonardo García Allende http://orcid.org/0000-0001-52372840

\section{REFERENCES}

1. Mazón A, Gil-Setas A, Sota de la Gándara LJ, Vindel A, Sáez-Nieto JA. Transmission of Streptococcus pyogenes causing successive infections in a family. Clin Microbiol Infect 2003;9(6): 554-559.

2. Lopardo HA, Vidal P, Sparo M, Jeric P, Centron D, Facklam RR, et al. Six-month multicenter study on invasive infections due to Streptococcus pyogenes and Streptococcus dysgalactiae subsp. equisimilis in Argentina. J Clin Microbiol 2005;43(2): 802-807.

3. Pichichero ME, Casey JR. Defining and dealing with carriers of group A streptococci. Contemp Pediatr 2003;20(1):46-53.

4. Desjardins M, Delgaty KL, Ramotar K, Seetaram C, Toye B. Prevalence and mechanisms of erythromycin resistance in group A and group B Streptococcus: implications for report- ing susceptibility results. J Clin Microbiol 2004;42(12):56205623.

5. Mc Murry LM, Levy SB. Tetracycline resistance in gram-positive bacteria. In: Fischetti VA, editor. Gram-positive pathogens. Washington, DC: ASM Press; 2006, p. 660-677.

6. Lopardo HA, Hernandez C, Vidal P, Vazquez M, Rosaenz L, Rubinstein G, et al. Erythromycin-resistant Streptococcus pyogenes in Argentina. Medicina (B Aires) 2004;64(2):143-145.

7. Carapetis JR, Steer AC, Mulholland EK, Weber M. The global burden of group A streptococcal diseases. Lancet Infect Dis 2005;5(11):685-694.

8. Clinical and Laboratory Standards Institute. M100-S23: performance standards for antimicrobial susceptibility testing; twenty-third informational supplement; 2013 [cited 2017 Mar 3]. Available from: file:///C:/Users/user/Downloads/CLSI\%20 2013.pdf.

9. Takeuchi T, Kawakita S. A follow-up study of throat carriers of streptococci among schoolchildren in Otsu City. Jpn Circ J 1985;49(12):1254-1257.

10. National Committee of Pediatric Infectology. Blue book of pediatric infectology. Buenos Aires: Argentine Society of Pediatrics; 1998, p. 513-515 (Spanish).

11. Kreikemeyer B, Klenk M, Podbielski A. The intracellular status of Streptococcus pyogenes: role of extracellular matrix-binding proteins and their regulation. Int J Med Microbiol 2004; 294(2-3):177-188.

12. Benouda A, Sibile S, Ziane Y, Elouennass M, Dahani K, Hassani A. Place of Streptococcus pyogenes in the throat infection in Morocco and overview of its susceptibility to antibiotics. Pathol Biol (Paris) 2009;57(1):76-80 (French).

13. Tellechea AL, Salvo MG, Méndez JH, Cavagnari BM. Group A beta-hemolytic Streptococcus frequency in the throat of symptomatic patients younger than 15 years, by age group. Arch Argent Pediatr 2012;110(6):516-519 (Spanish).

14. Gracia M, Díaz C, Coronel P, Gimeno M, García-Rodas R, Rodríguez-Cerrato V, et al. Antimicrobial susceptibility of Streptococcus pyogenes in Central, Eastern, and Baltic European countries, 2005 to 2006: the cefditoren surveillance program. Diagn Microbiol Infect Dis 2009;64(1):52-56.

15. Lopardo HA, Hernandez C, Vidal P. Antimicrobial resistance in Streptococcus pyogenes. An eleven-year surveillance in a pediatric hospital from Buenos Aires. Acta Bioquim Clin Latinoam 2004;38(2):151-157 (Spanish).

16. Liu X, Shen X, Chang H, Huang G, Fu Z, Zheng Y, et al. High macrolide resistance in Streptococcus pyogenes strains isolat- 
ed from children with pharyngitis in China. Pediatr Pulmonol 2009;44(5):436-441.

17. Nielsen HU, Hammerum AM, Ekelund K, Bang D, Pallesen LV, Frimodt-Møller N. Tetracycline and macrolide co-resistance in Streptococcus pyogenes: co-selection as a reason for increase in macrolide-resistant S. pyogenes? Microb Drug Resist 2004; 10(3):231-238.

18. Ayer V, Tewodros W, Manoharan A, Skariah S, Luo F, Bessen DE. Tetracycline resistance in group a streptococci: emergence on a global scale and influence on multiple-drug resistance. An- timicrob Agents Chemother 2007;51(5):1865-1868.

19. Feng L, Lin H, Ma Y, Yang Y, Zheng Y, Fu Z, et al. Macrolide-resistant Streptococcus pyogenes from Chinese pediatric patients in association with Tn916 transposons family over a 16year period. Diagn Microbiol Infect Dis 2010;67(4):369-375.

20. Bingen E, Bidet $P$, Mihaila-Amrouche $L$, Doit $C$, Forcet $S$, Brahimi N, et al. Emergence of macrolide-resistant Streptococcus pyogenes strains in French children. Antimicrob Agents Chemother 2004;48(9):3559-3562. 